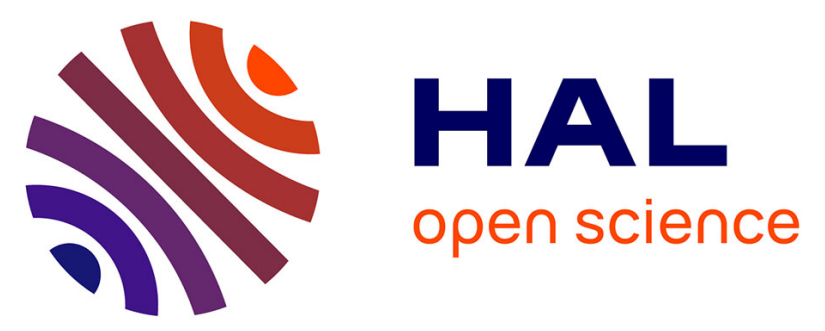

\title{
Reduction of Gordon-Mollenauer phase noise in dispersion-manged systems using in-line spectral inversion
}

David Boivin, Gee-Kung Chang, John R. Barry, Marc Hanna

\section{To cite this version:}

David Boivin, Gee-Kung Chang, John R. Barry, Marc Hanna. Reduction of Gordon-Mollenauer phase noise in dispersion-manged systems using in-line spectral inversion. Journal of the Optical Society of America B, 2006, 23 (10), pp.2019-2023. 10.1364/JOSAB.23.002019 . hal-00872137

HAL Id: hal-00872137

https://hal-iogs.archives-ouvertes.fr/hal-00872137

Submitted on 11 Oct 2013

HAL is a multi-disciplinary open access archive for the deposit and dissemination of scientific research documents, whether they are published or not. The documents may come from teaching and research institutions in France or abroad, or from public or private research centers.
L'archive ouverte pluridisciplinaire HAL, est destinée au dépôt et à la diffusion de documents scientifiques de niveau recherche, publiés ou non, émanant des établissements d'enseignement et de recherche français ou étrangers, des laboratoires publics ou privés. 


\title{
Reduction of Gordon-Mollenauer phase noise in dispersion-managed systems using in-line spectral inversion
}

\author{
David Boivin, Gee-Kung Chang, and John R. Barry \\ School of Electrical and Computer Engineering, Georgia Institute of Technology, Atlanta, Georgia 30318
}

Marc Hanna

Laboratoire Charles Fabry de l'Institut d'Optique, du Centre National de la Recherche Scientifique et de l'Université Paris Sud, Centre Universitaire, Bâtiment 503, 91403 Orsay Cedex, France

\begin{abstract}
Received March 30, 2006; revised May 31, 2006; accepted June 3, 2006; posted June 30, 2006 (Doc. ID 69432)
The influence of spectral inversion on the phase jitter of a soliton propagating in single-channel arbitrary dispersion-managed systems is studied with a semianalytic moment method. The results are similar to those previously observed in constant-dispersion links and show that the transmission-system reach can significantly be increased. (C) 2006 Optical Society of America
\end{abstract}

OCIS codes: $060.0060,060.1660,060.5060,060.5530$.

\section{INTRODUCTION}

Differential phase-shift-keying (DPSK) modulation formats have been studied intensively in the past few years. ${ }^{1}$ They were shown to outperform their intensitymodulated counterparts on key points in wavelengthdivision-multiplexed systems: high spectral efficiency, better tolerance to nonlinear impairments and cascaded filtering, and a $3 \mathrm{~dB}$ higher sensitivity when combined with balanced detection. On the other hand, DPSK formats are subject to impairments that are not usually encountered in intensity-modulated systems. Long-haul transmission systems using DPSK are primarily corrupted by nonlinear phase jitter because power fluctuations arising from amplified spontaneous emission (ASE) noise are translated into phase fluctuations through the self-phase modulation (SPM) effect. ${ }^{2}$ Among the techniques that have been proposed ${ }^{3,4}$ and later developed ${ }^{5,6}$ to limit this nonlinear amplitude-to-phase-noise conversion also known as the Gordon-Mollenauer effect, spectral inversion (SI) or optical phase conjugation promised to be effective. ${ }^{7}$ In lossless constant-dispersion links, midspan spectrum inversion makes the phase-conjugate signal experience opposite dispersion and nonlinearity in the second half of the link, providing an exact compensation. ${ }^{8}$ Even if nonuniform distributed amplification and dispersion management (DM) break the power symmetry required for exact compensation, nonlinearity control is still observed in conventional transmission lines. ${ }^{9,10}$

Here we present the first evaluation, to our knowledge, of phase jitter in single-channel DM soliton (DMS) links using SI. This calculation is based on a semianalytic moment method. We make the same set of assumptions as in Ref. 11; i.e., the chirp remains linear, and the optical filters are Gaussian shaped. Our method, which requires a single numerical resolution of the propagation equation, is validated by comparison with Monte Carlo simulations. The excellent agreement showed by the results provides a fast estimate for the extent of the transmission reach and insight into the physical mechanisms that reduce phase jitter in such systems.

\section{DERIVATION}

The propagation of a single optical pulse envelope $u(z, t)$ in the transmission line is governed by the nonlinear Schrödinger equation (NLSE). Given that the propagation of the phase-conjugated field $u^{*}$ in the second half of the link is equivalent to changing the sign of the local groupvelocity dispersion $\beta_{2}$ and nonlinearity $\gamma,{ }^{8}$ the NLSE can be generalized to take SI into account:

$$
i \frac{\partial u}{\partial z}-\frac{1}{2}\left[\epsilon \beta_{2}(z)-i b(z)\right] \frac{\partial^{2} u}{\partial t^{2}}+\epsilon \gamma(z)|u|^{2} u=i g(z) u+F(z, t),
$$

where $g(z)$ is the local net gain and $b(z)$ is the filtering factor. When $\epsilon$ evaluates to 1, Eq. (1) describes the standard propagation of the optical field before SI. When $\epsilon$ evaluates to -1, Eq. (1) describes the propagation of the phaseconjugated field after SI. The noise source term $F$ is described by its autocorrelation function ${ }^{12}$ :

$$
\left\langle F(z, t) F^{*}\left(z^{\prime}, t^{\prime}\right)\right\rangle=2 g_{0} \hbar \omega_{0} n_{\mathrm{sp}}(z) \delta\left(z-z^{\prime}\right) \delta\left(t-t^{\prime}\right),
$$

where $n_{\mathrm{sp}}$ is the spontaneous emission factor, $g_{0}$ is the amplification coefficient inside the amplifier, and $\hbar \omega_{0}$ is the photon energy at the signal frequency. The factor 2 in Eq. (2) accounts for the unpolarized nature of ASE.

We define the following quantities averaged over time: 


$$
\begin{aligned}
& E=\int_{-\infty}^{+\infty}|u|^{2} \mathrm{~d} t, \\
& P=\frac{1}{E} \int_{-\infty}^{+\infty}|u|^{4} \mathrm{~d} t, \\
& \Phi=\frac{1}{E} \int_{-\infty}^{+\infty}|u|^{2} \arg (u) \mathrm{d} t .
\end{aligned}
$$

The quantities $E, P$, and $\Phi$ respectively represent the energy, power, and phase of the optical field at a given distance. By differentiating $\Phi$ and $P$ with respect to $z$ and using the generalized NLSE (1) under the assumption that the pulse mostly exhibits linear chirp, i.e., $\arg (u)(z, t)$ $\approx \phi_{0}(z)+\phi_{2}(z)\left(t-t_{0}\right)^{2}$, we derived a further generalized dynamic system:

$$
\begin{aligned}
\frac{\mathrm{d} \Phi}{\mathrm{d} z}= & -\epsilon \beta_{2} \phi_{2} \Phi+\epsilon \gamma P+\frac{i}{E} \int_{-\infty}^{+\infty}[\arg (u)-\Phi]\left(u \hat{F}^{*}-u^{*} \hat{F}\right) \mathrm{d} t \\
& -\frac{1}{2 E} \int_{-\infty}^{+\infty} u^{*} \hat{F}+u \hat{F}^{*} \mathrm{~d} t, \\
\frac{\mathrm{d} P}{\mathrm{~d} z}= & 2\left[g+\epsilon \beta_{2} \phi_{2}-\frac{b(z)}{2 E} \int_{-\infty}^{+\infty}\left|u_{t}\right|^{2} \mathrm{~d} t\right] P+\frac{i}{E} \int_{-\infty}^{+\infty}\left(2|u|^{2}-P\right) \\
& \times\left(u \hat{F}^{*}-u^{*} \hat{F}\right) \mathrm{d} t .
\end{aligned}
$$

These equations describe the evolution of the moments $\Phi$ and $P$ along the optical link.

\section{A. Evolution before Spectral Inversion}

Here we recall the results obtained for filtered systems in Ref. 11.

In the case where $\epsilon=1$, we can implicitly integrate the dynamic equations that govern the evolution of power and phase by using the method of variation of parameters ${ }^{13}$ :

$$
\begin{aligned}
P(z)= & \left(P_{0}+i \int_{0}^{z}\left\{\frac { 1 } { E A _ { 1 } } \int _ { - \infty } ^ { + \infty } [ ( 2 | u | ^ { 2 } - P ) ] \left(u \hat{F}^{*}\right.\right.\right. \\
& \left.\left.\left.-u^{*} \hat{F}\right) \mathrm{~d} t\right\} \mathrm{~d} z_{1}\right) A_{1},
\end{aligned}
$$

where

$$
\begin{aligned}
A_{1}(z) & =\exp \left\{\int_{0}^{z} 2\left[g+\beta_{2} \phi_{2}-\frac{b(z)}{2 E} \int_{-\infty}^{+\infty}\left|u_{t}\right|^{2} \mathrm{~d} t\right] \mathrm{d} z_{1}\right\}, \\
\Phi & =\Phi_{1}+\Phi_{2}+\Phi_{3}
\end{aligned}
$$

where

$$
\Phi_{1}=A_{2} \int_{0}^{z} \frac{\gamma P}{A_{2}} \mathrm{~d} z_{1},
$$

$$
\begin{aligned}
\Phi_{2}= & i A_{2} \int_{0}^{z}\left\{\frac{1}{E A_{2}} \int_{-\infty}^{+\infty}[\arg (u)-\Phi]\right. \\
& \left.\times\left(u \hat{F}^{*}-u^{*} \hat{F}\right) \mathrm{d} t\right\} \mathrm{d} z_{1}, \\
\Phi_{3}= & -\frac{A_{2}}{2} \int_{0}^{z}\left[\frac{1}{E A_{2}} \int_{-\infty}^{+\infty}\left(u^{*} \hat{F}+u \hat{F}^{*}\right) \mathrm{d} t\right] \mathrm{d} z_{1}, \\
A_{2}(z)= & \exp \left(-\int_{0}^{z} \beta_{2} \phi_{2} \mathrm{~d} z_{1}\right) .
\end{aligned}
$$

The phase variance can be calculated using Eqs. (2), (8), and (10):

$$
\sigma_{\Phi}^{2}=\left\langle\Phi^{2}\right\rangle-\langle\Phi\rangle^{2}=\left\langle\Phi_{1}^{2}\right\rangle+\left\langle\Phi_{2}^{2}\right\rangle+\left\langle\Phi_{3}^{2}\right\rangle+2\left\langle\Phi_{1} \Phi_{2}\right\rangle,
$$

where

$$
\left\langle\Phi_{1}^{2}\right\rangle=A_{2}^{2} \int_{0}^{z} \frac{\gamma A_{1}}{A_{2}} \int_{0}^{z_{1}} \frac{\gamma A_{1}}{A_{2}} \int_{0}^{z_{2}}\left(\delta q_{1}, \delta q_{1}\right) \mathrm{d} z_{3} \mathrm{~d} z_{2} \mathrm{~d} z_{1},
$$

$$
\begin{gathered}
\left\langle\Phi_{2}^{2}\right\rangle=\frac{A_{2}^{2}}{2} \int_{0}^{z}\left(\delta q_{2}, \delta q_{2}\right) \mathrm{d} z_{1}, \\
\left\langle\Phi_{3}^{2}\right\rangle=\frac{A_{2}^{2}}{8} \int_{0}^{z}\left(\delta q_{3}, \delta q_{3}\right) \mathrm{d} z_{1},
\end{gathered}
$$

$$
2\left\langle\Phi_{1} \Phi_{2}\right\rangle=A_{2}^{2} \int_{0}^{z} \gamma A_{1} \int_{0}^{z_{1}}\left(\delta q_{1}, \delta q_{2}\right) \mathrm{d} z_{2} \mathrm{~d} z_{1},
$$

defining the scalar product

$$
\left(\delta q_{i}, \delta q_{j}\right)=\int_{-\infty}^{+\infty} \delta q_{i} \delta q_{j}^{*}+\delta q_{i}^{*} \delta q_{j} \mathrm{~d} t
$$

and the functions

$$
\begin{aligned}
& \delta q_{1}=2 i \sqrt{g n_{\mathrm{sp}} \hbar \omega_{0}} \frac{2|u|^{2}-P}{E A_{1}} u, \\
& \delta q_{2}=2 i \sqrt{g n_{\mathrm{sp}} \hbar \omega_{0}} \frac{\arg (u)-\Phi}{E A_{2}} u,
\end{aligned}
$$

$$
\delta q_{3}=2 \sqrt{g n_{\mathrm{sp}} \hbar \omega_{0}} \frac{u}{E A_{2}} .
$$

The quantity $\left\langle\Phi_{1}^{2}\right\rangle$ has been identified ${ }^{11,13}$ as the phase jitter induced by power fluctuations through SPM or nonlinear phase jitter, $\left\langle\Phi_{2}^{2}\right\rangle$ and $\left\langle\Phi_{3}^{2}\right\rangle$ are direct contributions from the noise to phase jitter, and $\left\langle\Phi_{1} \Phi_{2}\right\rangle$ is the interference term between these two effects.

\section{B. Evolution after Spectral Inversion}

The main result presented here concerns the propagation after SI where now $\epsilon=-1$. This sign change does not affect the direct contribution of noise to the phase variance, 
since linear phase kicks introduced at each amplifier are independent of one another. On the other hand, the nonlinear phase kicks are related to the peak power at the location of the amplifier, which is correlated to the previous values of peak powers. This, in turn, leads to correlation between nonlinear phase kicks. As a result, changing the sign of $\gamma$ introduces some anticorrelation between nonlinear phase kicks before and after SI, reducing the overall phase noise. From this observation, the only contribution to phase jitter that needs to be fully reevaluated when operating in the phase-conjugated section of the link is the nonlinear part of the phase.

The formal form of any parameter $X(z)$ expressed before SI can also be used after SI if one shifts the origin to the spectral inverter location $\left(z=L_{1}\right)$ and reverses the sign of $\beta_{2}$ and $\gamma . \hat{X}(z)$ will then refer to the evolution of the parameter $X$ after SI in the new coordinate system. Applying this to the nonlinear phase $\Phi_{1}$ gives its expression $\hat{\Phi}_{1}$ after SI:

$$
\hat{\Phi}_{1}(z)=\Phi_{1}\left(L_{1}\right)-\hat{A}_{2} \int_{0}^{z} \frac{\gamma \hat{P}\left(z_{1}\right)}{\hat{A}_{2}} \mathrm{~d} z_{1},
$$

with power fluctuations given by

$$
\begin{aligned}
\hat{P}(z)= & \hat{A}_{1}\left(P\left(L_{1}\right)+i \int_{0}^{z}\left\{\frac { 1 } { \hat { E } \hat { A } _ { 1 } } \int _ { - \infty } ^ { + \infty } [ ( 2 | u | ^ { 2 } - \hat { P } ) ] \left(u F^{*}\right.\right.\right. \\
& \left.\left.\left.-u^{*} F\right) \mathrm{~d} t\right\} \mathrm{~d} z_{1}\right),
\end{aligned}
$$

with

$$
\begin{aligned}
& \hat{A}_{1}(z)=\exp \left\{\int_{0}^{z} 2\left[g-\beta_{2} \phi_{2}-\frac{b(z)}{2 E} \int_{-\infty}^{+\infty}\left|u_{t}\right|^{2} \mathrm{~d} t\right] \mathrm{d} z_{1}\right\}, \\
& \hat{A}_{2}(z)=\exp \left(\int_{0}^{z} \beta_{2} \phi_{2} \mathrm{~d} z_{1}\right) .
\end{aligned}
$$

The total phase variance due to the Gordon-Mollenauer effect is then given by

$$
\left\langle\hat{\Phi}_{1}^{2}\right\rangle=\left\langle\Phi_{1}\left(L_{1}\right)^{2}\right\rangle+\sigma_{\Phi_{\mathrm{PC}_{1}}}^{2}(z)-\sigma_{\Phi_{\mathrm{PC}_{2}}}^{2}(z) .
$$

The first term, computed from Eq. (16), accounts for the phase jitter accumulated before the SI, which effect is further analyzed. The nonlinearity control provided in the second half of the link is materialized by the negative term in Eq. (28), which forces the total phase variance to increase at a slower rate. The natural tendency observed before conjugation is still observed in $\sigma_{\Phi_{\mathrm{PC}_{1}}}^{2}$, but its impact is limited by $\sigma_{\Phi_{\mathrm{PC}_{2}}}^{2}$. These contributions are given by

$$
\begin{aligned}
\sigma_{\Phi_{\mathrm{PC}_{1}}}^{2}(z)= & G(z)\left[\int_{0}^{z}\left(\delta \hat{q}_{1}, \delta \hat{q}_{1}\right) \mathrm{d} z_{3}+A_{1}^{2}\left(L_{1}\right) \int_{0}^{L_{1}}\left(\delta q_{1}, \delta q_{1}\right) \mathrm{d} z_{3}\right. \\
& \left.+2 A_{1}\left(L_{1}\right) \int_{0}^{\min \left(z, L_{1}\right)}\left(\delta q_{1}^{\prime}, \delta q_{1}^{\prime}\right) \mathrm{d} z_{3}\right]
\end{aligned}
$$

$$
\begin{aligned}
\sigma_{\Phi_{\mathrm{PC}_{2}}}^{2}(z)= & -\frac{3}{8} H(z)\left[A_{1}\left(L_{1}\right) A_{2}\left(L_{1}\right) \int_{0}^{L_{1}}\left(\delta q_{1}, \delta q_{1}\right) \mathrm{d} z_{3}\right. \\
& \left.+A_{2}\left(L_{1}\right) \int_{0}^{\min \left(z, L_{1}\right)}\left(\delta q_{1}^{\prime}, \delta q_{1}^{\prime}\right) \mathrm{d} z_{2}\right]
\end{aligned}
$$

The functions $\mathrm{G}, \mathrm{H}, \delta q_{1}, \delta \hat{q}_{1}$, and $\delta q_{1}^{\prime}$ are given by

$$
\begin{aligned}
& G(z)=\hat{A}_{2}^{2}(z) \int_{0}^{z} \frac{\gamma \hat{A}_{1}}{\hat{A}_{2}}\left(z_{1}\right) \int_{0}^{z_{1}} \frac{\gamma \hat{A}_{1}}{\hat{A}_{2}}\left(z_{2}\right) \mathrm{d} z_{2} \mathrm{~d} z_{1}, \\
& H(z)=\hat{A}_{2}(z) \int_{0}^{L_{1}} \frac{\gamma A_{1}}{A_{2}}\left(z_{2}\right) \mathrm{d} z_{2} \int_{0}^{z} \frac{\gamma \hat{A}_{1}}{\hat{A}_{2}}\left(z_{3}\right) \mathrm{d} z_{3}, \\
& \delta q_{1}=2 i \sqrt{g n_{\mathrm{sp}} \hbar \omega_{0}} \frac{2|u|^{2}-P}{E A_{1}} u, \\
& \delta \hat{q}_{1}=2 i \sqrt{g n_{\mathrm{sp}} \hbar \omega_{0}} \frac{2|u|^{2}-\hat{P}}{\hat{E} \hat{A_{1}}} u,
\end{aligned}
$$

$$
\delta q_{1}^{\prime}=2 i \sqrt{g n_{\mathrm{sp}} \hbar \omega_{0}} \frac{2|u|^{2}-\hat{P}}{E \sqrt{A_{1} \hat{A}_{1}}} u .
$$

$G(z)$ and $H(z)$ are the dominant functions that determine the evolution of the phase variance after the conjugation. Their relative variations explain the existence of a distance for which phase jitter is minimum. Equations (28)-(35) represent the main result of this paper and are valid for arbitrary pulse shapes and single-channel communication systems, provided that the chirp remains essentially linear throughout the propagation. The effects of intrachannel interactions remain to be studied in depth.

\section{SIMULATIONS AND DISCUSSION}

To demonstrate the validity of the moment method, we apply it to DM soliton systems with different levels of filter strength and check our model by comparing it with direct Monte Carlo simulations. The average phase of the field was numerically evaluated over an ensemble of 512 simulations with different ASE noise used to infer the phase variance.

The optical links are formed by one's alternating $30 \mathrm{~km}$ spans of anomalous dispersion fiber with $D_{1}$ $=11 \mathrm{ps} /(\mathrm{nm} / \mathrm{km})$ and $A_{\text {eff }}=50 \mu \mathrm{m}^{2}$ and normal dispersion fiber with $D_{2}=-10 \mathrm{ps} /(\mathrm{nm} / \mathrm{km})$ and $A_{\text {eff }}=50 \mu \mathrm{m}^{2}$. The $\mathrm{DM}$ soliton link operates at a high peak power $(7.2 \mathrm{~mW})$. Unchirped pulses are launched at the midpoint of the normal dispersion span. Optical amplifiers followed by Gaussian filters are placed every $z_{a}=60 \mathrm{~km}$ along the link, with a noise factor $n_{\mathrm{sp}}=1.5$. The full width at halfmaximum bandwidth $B$ of the filters in terms of optical power is related to the filtering factor by the relation $b$ $=\ln (2) /\left(\pi^{2} B^{2} z_{a}\right)$.

The standard deviation of the soliton phase is plotted in Fig. 1 for different filter bandwidths with and without midlink SI. As the signal bandwidth is about $15 \mathrm{GHz}$, the 
$500 \mathrm{GHz}$ filter case corresponds to an almost unfiltered system. We observe that the phase standard deviation is greatly reduced by SI. This reduction is less effective when tight filtering is used. This can be explained by noting that the amount of nonlinear phase noise accumulated in the first half of the link is less important in that case. Nevertheless, midlink SI enables unfiltered systems to show the same performance after $6000 \mathrm{~km}$ as standard $100 \mathrm{GHz}$ systems. We also note a satisfactory agreement between the results of the moment method and those of the Monte Carlo simulations.

The phase variance $\left\langle\Phi_{1}^{2}\right\rangle$ resulting from the nonlinear phase noise mediated by SPM is plotted in Fig. 2 for the case of $100 \mathrm{GHz}$ filters. Its different contributions (shown in dashed curves) after SI directly reflect the dominant behavior of the functions $G(z)$ and $H(z)$ defined in Eqs. (31) and (32). Their relative variations modify the overall evolution in a way similar to the constant-dispersion soliton case ${ }^{7}$ in which nonlinearity control is achieved and results in the existence of a minimum value for the phase jitter. Since this minimum is reached somewhere in the second half-part of the propagation, midlink SI is certainly not optimal. This leads us to study the impact of the SI location. Figure 3 depicts the evolution of the standard deviation of the phase as a function of distance when the location of the spectral inverter is changed from its

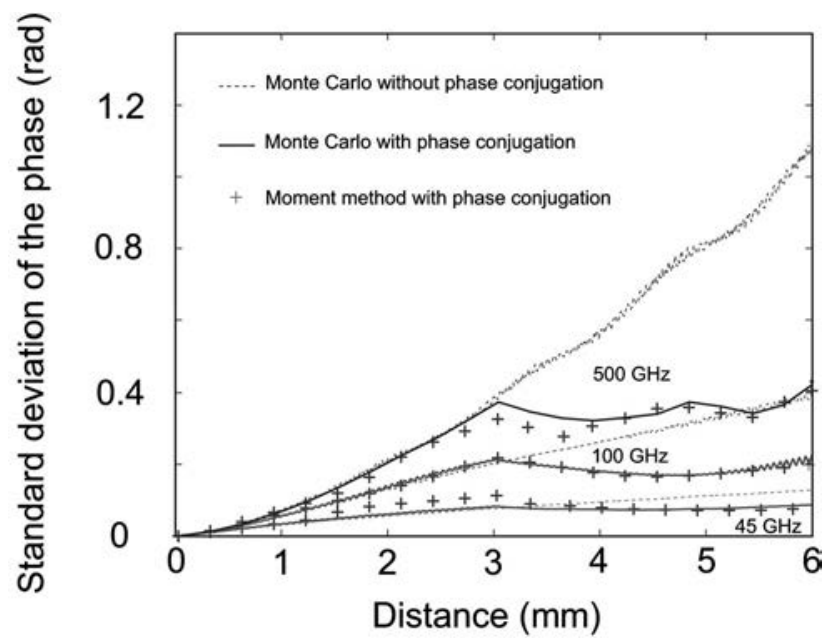

Fig. 1. Standard deviation of the phase as a function of distance for the DM soliton system with and without SI.

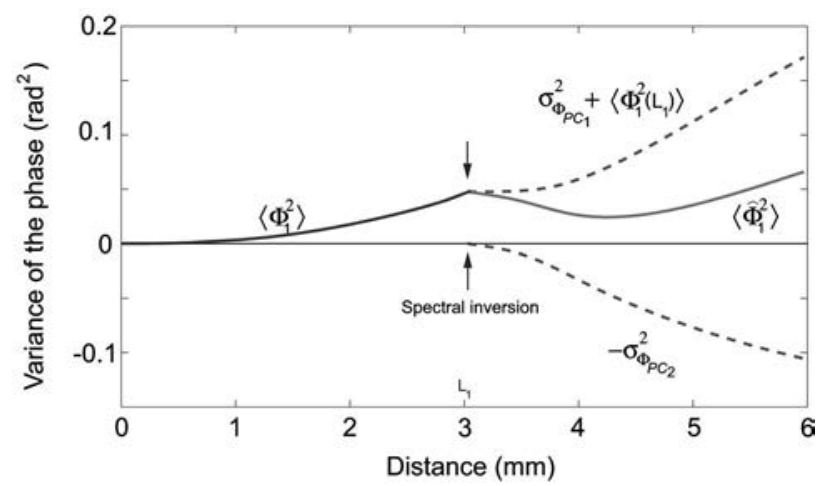

Fig. 2. Variance of the nonlinear phase as a function of distance for the $100 \mathrm{GHz}$ DMS system.

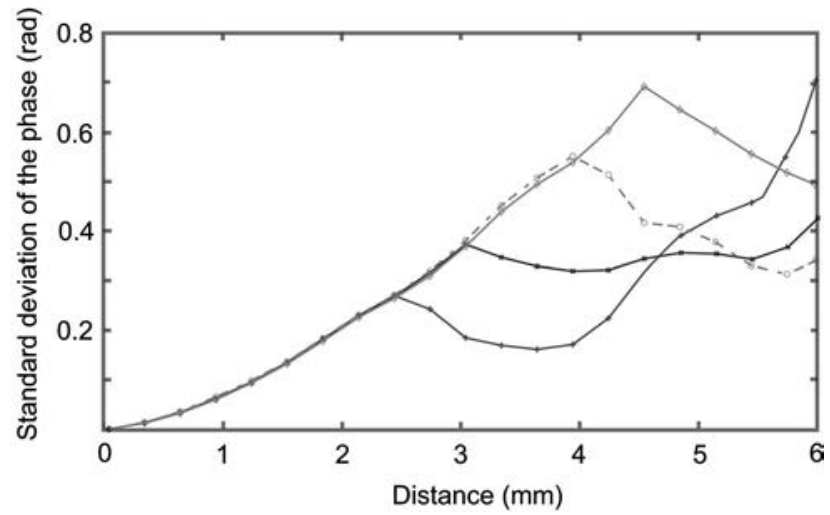

Fig. 3. Phase variance evolution as a function of the spectral inverter location for the $100 \mathrm{GHz}$ DMS system.

symmetrical position $\left(L_{1}=3000 \mathrm{~km}\right)$ to 2500,4000 , and $4500 \mathrm{~km}$. The latter two cases correspond to a two-thirdand three-fourths-link SI.

First, we can see that, if SI is performed before midlink, we rapidly lose the benefit of nonlinear compensation, since we observe a growth similar to the one observed without SI for the same system in Fig. 1. This can be explained by the fact that, after $L_{1} \mathrm{~km}$ propagation in the second half of the link, Eq. (30) has a different behavior. In that case, $\min \left(z, L_{1}\right)=L_{1}$, which means that the multiplicative term modulating $H(z)$ is now constant. If the same conclusion can be drawn for the analog term in Eq. (29), the overall multiplicative coefficient in front of $G(z)$ is still dependent on $z$, which leads to a nonlinear phase jitter evolution similar to the one before SI. The similarity is also reflected in the notations, since the driving term of $\sigma_{\Phi_{\mathrm{PC}_{1}}}^{2}-\sigma_{\Phi_{\mathrm{PC}_{2}}}^{2}$ is $G(z) \int_{0}^{z}\left(\delta \hat{q}_{1}, \delta \hat{q}_{1}\right) \mathrm{d} z_{3}$ and can be compared with its counterpart before SI expressed by Eq. (16). Intuitively, the potential correlations between phase kicks before and after SI cannot extend beyond a propagation distance of $2 L_{1}$.

Finally, when SI is performed after the midlink point, the results show that the best location is close to the twothirds link. In this quasi-optimal case, the maximum nonlinear phase jitter control is achieved just before $6000 \mathrm{~km}$. The performance reached in terms of standard deviation in this case results in a 2.11 range extension when compared with the extent of the corresponding link without SI to get the same performance.

\section{CONCLUSION}

We have shown that in-line spectral inversion is an effective technique to reduce nonlinear phase jitter in singlechannel DMS systems. Because phase fluctuations are not Gaussian, the model based on the moment method developed here cannot be expected to accurately predict exact bit error rates. ${ }^{14,15}$ Nevertheless, it quickly evaluates phase jitter in arbitrary dispersion-managed systems, and its good agreement with Monte Carlo simulations makes it a useful and practical tool to qualitatively assess the performance of DPSK systems. As phase conjugation compensates for nonlinearity, cross-phase-modulationinduced phase noise reduction is also expected in multichannel systems. ${ }^{16}$ 
D. Boivin, the corresponding author, can be reached by e-mail at david.boivin@gatech.edu.

\section{REFERENCES}

1. A. H. Gnauck and P. J. Winzer, "Optical phase-shift-keyed transmissions," J. Lightwave Technol. 23, 115-130 (2005).

2. J. P. Gordon and L. F. Mollenauer, "Phase noise in photonic communications systems using linear amplifiers," Opt. Lett. 15, 1351-1353 (1990).

3. X. Liu, X. Wei, R. E. Slusher, and C. J. McKinstrie, "Improving transmission performance in differential phaseshift-keyed systems by use of lumped nonlinear phase-shift compensation," Opt. Lett. 27, 1616-1618 (2002).

4. C. Xu and X. Liu, "Postnonlinearity compensation with data-driven phase modulators in phase-shift keying transmission," Opt. Lett. 27, 1619-1621 (2002).

5. J. Hansryd, J. van Howe, and C. Xu, "Experimental demonstration of nonlinear phase jitter compensation in DPSK modulated fiber links," IEEE Photon. Technol. Lett. 17, 232-234 (2005).

6. M. Matsumoto, "Performance improvement of phase-shiftkeying signal transmission by means of optical limiters using four-wave mixing in fibers," J. Lightwave Technol. 23, 2696-2701 (2005).

7. C. J. McKinstrie, S. Radic, and C. Xie, "Reduction of soliton phase jitter by in-line phase conjugation," Opt. Lett. 28, 1519-1521 (2003).

8. G. P. Agrawal, Fiber-Optic Communication Systems (Wiley, 2002).

9. A. Chowdhury, G. Raybon, R.-J. Essiambre, J. Sinsky, A. Adamiecki, J. Leuthold, C. R. Doerr, and S.
Chandrasekhar, "Compensation of intra-channel nonlinearities in $40 \mathrm{~Gb} / \mathrm{s}$ pseudo-linear systems using optical phase conjugation," in Optical Fiber Communication Conference (OFC), Vol. 95 of OSA Trends in Optics and Photonics Series (Optical Society of America, 2004), paper PDP32.

10. S. L. Jansen, D. van den Borne, C. Climent Monsalve, S. Spälter, P. M. Krummrich, G. D. Khoe, and H. de Waardt, "Reduction of Gordon-Mollenauer phase noise by midlink spectral inversion," IEEE Photon. Technol. Lett. 17, 923-925 (2005).

11. D. Boivin, M. Hanna, P.-A. Lacourt, and J.-P. Goedgebuer, "Reduction of phase jitter in dispersion-managed systems by in-line filtering," Opt. Lett. 29, 688-690 (2004).

12. H. A. Haus, "Quantum noise in a solitonlike repeater system,” J. Opt. Soc. Am. B 8, 1122-1126 (1991).

13. M. Hanna, D. Boivin, P.-A. Lacourt, and J.-P. Goedgebuer, "Calculation of optical phase jitter in dispersion-managed systems by use of the moment method," J. Opt. Soc. Am. B 21, 24-28 (2003).

14. E. T. Spiller, W. L. Kath, R. O. Moore, and C. J. McKinstrie, "Computing large signal distortions and bit-error ratios in DPSK transmission systems," IEEE Photon. Technol. Lett. 17, 1022-1024 (2005)

15. E. T. Spiller, G. M. Donovan, and W. L. Kath, "Direct determination of range extension due to phase conjugation in a soliton-based DPSK transmission system," in Conference on Lasers and Electro-Optics (CLEO), OSA Trends in Optics and Photonics Series (Optical Society of America, 2005), paper CWO4.

16. C. J. McKinstrie, C. Xie, and C. Xu, "Effects of cross-phase modulation on phase jitter in soliton systems with constant dispersion," Opt. Lett. 28, 604-606 (2003). 